\title{
Emergency Department Crowding is Harder to Solve: The Impact of COVID-19 of Longer Length of Stay
}

\section{Fengbao Guo}

the First Affiliated Hospital of Soochow University

Yan Qin

the First Affiliated Hospital of Soochow University

Hailong Fu

the First Affiliated Hospital of Soochow University

Feng Xu ( $\sim$ qy15704900135@163.com)

the First Affiliated Hospital of Soochow University

\section{Research Article}

Keywords: COVID-19, Length Of Stay, Emergency department crowding

Posted Date: December 28th, 2021

DOI: https://doi.org/10.21203/rs.3.rs-1089883/v1

License: (c) (i) This work is licensed under a Creative Commons Attribution 4.0 International License.

Read Full License 


\section{Abstract \\ Objectives}

To determine the impact of the Coronavirus disease-2019 (COVID-19) pandemic on the length of stay (LOS) and prognosis of patients in the emergency department (ED).

\section{Methods}

A retrospective review of case data of patients in the ED during the early stages of the COVID-19 pandemic in the First Affiliated Hospital of Soochow University (January 15, 2020 - January 14, 2021) was performed and compared with that during the pre-COVID-19 period (January 15, 2019 - January 14, 2020). Patient information including age, sex, length of stay, and death was collected. Wilcoxon Rank sum test was utilized to compare the difference in LOS between the two cohorts. Chi-Squared test was utilized to analyze the prognosis of patients. The LOS and prognosis in different departments (emergency internal medicine, emergency surgery, emergency neurology, and other departments) were further analyzed.

\section{Results}

Of the total 8278 patients, 4159 (50.24\%) were ordered in the COVID-19 pandemic group and 4119 (49.76\%) were ordered in the pre-COVID-19 group. The length of stay prolongs significantly in the COVID19 group compared with that in the pre-COVID-19 group(13h vs $9.8 \mathrm{~h} ; \mathrm{p}<0.001)$. There was no significant difference in mortality between the two cohorts (4.8\% VS 5.3\%; $p=0.341)$.

\section{Conclusion}

The COVID-19 pandemic was associated with a significant increase in the length of stay, which may lead to emergency department crowding. And the influence of the COVID-19 pandemic on patients in different emergency departments is different. There is no significant impact on the LOS of emergency neuropathy. Across departments, COVID-19 didn't have a significant impact on the prognosis of ED patients.

\section{Background}

COVID-19 is acute infectious pneumonia that was declared a global pandemic by WHO in 2020. [1, 2]Fatal cases ended in respiratory distress and lung failure. [3] It not only seriously harms human health, but also poses a challenge to international health causes. On January 15, 2020, the Chinese Center for Disease Control and Prevention (CDC) launched its first-level emergency response to a public health emergency. Medical and health institutions have actively implemented epidemic prevention and control plans and conducted a series of screening for hospitalized patients, which has played an important role 
in controlling the spread of the epidemic. The number of patients in ED is large and the mobility is strong, which increases the difficulty for emergency medical staff. The increased workload during COVID-19 could exacerbate the widespread and serious problems already existing in emergency departments around the world --ED Crowding and prolonged LOS in ED.

The ED was crowded because of the mismatch between ED capacity and the number of patients presenting in a given period and has become a serious public health problem.[4] Its definition is still unclear. [5]The causes for crowding in the ED can be summarized into three aspects: input, throughput, and output.[6-9]The prolonged length of stay (LOS) time of patients in the ED is one of the main reasons for ED crowding. ED crowding may increase the length of stay (LOS), delay the evaluation and treatment of patients, increase the work pressure of medical staff, increase the incidence of medical disputes and other adverse events. [10]

As a "window" of the hospital, it is essential to know whether the pandemic has prolonged the LOS in the ED or caused the crowding of the ED. It is significant to timely treat patients and effectively prevent the spread of the epidemic in a dense crowd. However, to our knowledge, there are few reports on whether the COVID-19 pandemic has caused prolonged LOS in ED. The purpose of this study was to determine the impact of the COVID-19 pandemic on the LOS and prognosis of ED patients. The significance of this study is providing basic data that can help public health systems respond to ED crowding during a public health emergency, facilitating future clinical research and improving patient outcomes.

\section{Methods}

\subsection{Study design}

A retrospective observational study was performed. Patient information, including age, sex, length of stay and death, through the ED at the First Affiliated Hospital of Soochow University were gathered during the period of COVID-19 pandemic (January 15, 2020 - January 14, 2021) and compared with the same duration in the pre-COVID-19 period (January 15, 2019 - January 14, 2020).

\subsection{Statistical analysis}

Continuous measurements with a normal distribution were summarized with means and standard deviations. Median and interquartile ranges (IQRs) were used to summarize continuous measurements without normal distribution. Categorical measurements were summarized using frequencies and percentages. A Ranksum test was performed to examine the difference in LOS pre-COVID-19 group and during the COVID-19 group. Chisquare tests were conducted between different months to address differences between the two-time points. Chisquare test or Fisher's exact test was used to examine the differences in the mortality rate in the pre-COVID-19 group and the COVID-19 group. Daily averages were calculated for all months to control for the sample size difference between each time frame. All statistical analyses were performed using $\mathrm{R}$ language version 4.1 .0 , and a $p<0.05$ was considered statistically significant. Assumption checks were performed prior to all analyses. 


\subsection{Ethics approval}

This retrospective cohort study was performed in accordance with the Declaration of Helsinki and approved by the Medical Ethics Committee of the First Affiliated Hospital of Soochow University. (2021) Research Lot No. 317

\section{Results}

Baseline demographic clinical characteristics in the ED patients are shown in Table 1. From January 2019 to January 2021, a total of 8278 patients were screened, with 4119 (49.76\%) pre-COVID-19 patients and 4159 (50.24\%) COVID-19 patients. There were no significant differences in age $(P=0.241)$ and gender $(P=0.468)$ between the two groups.

Table 1

Baseline demographic clinical characteristics in the ED patients of the pre-COVID-19 group and the COVID-19 group

\begin{tabular}{|llll|}
\hline & $\begin{array}{l}\text { pre-COVID-19 group } \\
(\mathbf{n = 4 1 1 9 )}\end{array}$ & $\begin{array}{l}\text { COVID-19 group } \mathrm{n}= \\
(\mathbf{4 1 5 9 )}\end{array}$ & $\begin{array}{l}\text { P } \\
\text { value }\end{array}$ \\
\hline Age, Mean(IQR)(years) & $64(48,75)$ & $64(49,76)$ & 0.241 \\
\hline Sex & & & 0.468 \\
\hline Male, $\mathrm{n}(\%)$ & $2564(62.2 \%)$ & $2621(63 \%)$ & \\
\hline Female, $\mathrm{n}(\%)$ & $1555(37.8 \%)$ & $1538(37 \%)$ & 0.715 \\
\hline Department & & & \\
\hline $\begin{array}{l}\text { Emergence internal medicine, } \mathrm{n} \\
\text { (\%) }\end{array}$ & $2500(60.7 \%)$ & $2516(60.5 \%)$ & \\
\hline Emergency surgery,n (\%) & $705(17.1 \%)$ & $696(16.7 \%)$ & $<0.001$ \\
\hline Emergency neurology, $\mathrm{n}(\%)$ & $842(20.4 \%)$ & $883(21.2 \%)$ & 0.341 \\
\hline Else, $\mathrm{n}(\%)$ & $72(1.7 \%)$ & $64(1.5 \%)$ & \\
\hline Los, Mean (h) & 9.8 & 13 & \\
\hline Mortality, $\mathrm{n}(\%)$ & $199(4.8 \%)$ & $220(5.3 \%)$ & \\
\hline
\end{tabular}

There were no obvious visual differences in the number of ED visits between the pre-COVID-19 group and the COVID-19 group in emergency internal medicine, emergency surgery, emergency neurology, and other emergency departments $(P=0.715)$. The LOS was significantly longer in the COVID-19 group than the preCOVID-19 group $(P<0.01)$ (Table 1). LOS median time (IQR) increased from $9.8 \mathrm{~h}(3,22)$ to13h $(3.5,23.9)$ $(p<0.001)$. Statistical analysis of LOS in each department (Fig. 1) showed that the LOS in the COVID-19 group was prolonged in both emergency internal medicine (15.3h VS 11.3h) and emergency surgery (8.7h VS $4.9 \mathrm{~h}$ ), especially in emergency surgery (8.7h VS $4.9 \mathrm{~h}$ ). However, the LOS of the emergency neurology 
department and other departments in the COVID-19 group was not statistically significant compared with the pre-COVID-19 group ( $P=0.106, P=0.084)$ LOS was statistically analyzed in each department by month, as shown in Figure 2. LOS increased significantly after the COVID-19 pandemic in the whole emergency department (Fig. 2, A), emergency internal medicine (Fig. 2, B), and emergency surgery (Fig. 2, C). However, there was no increasing trend in the LOS of the emergency neurology department (Fig. 2, D).

In this study, the number of deaths in the ED was 419 (220 in the COVID-19 group and 199 in the preCOVID-19 group). Although the LOS in the COVID-19 group was significantly longer than that in the preCOVID-19 group, there was no statistically significant relationship in the proportion of deaths between the two groups (5.3\% VS 4.8\%) $(P=0.341)$. And there was no difference in the proportion of deaths among subgroups, including emergency internal medicine ( $6 \%$ vs $6.7 \%, P=0.271)$, emergency surgery $(3.8 \%$ vs $3.6 \%, P=0.814)$, emergency neurology $(2.7 \%$ vs $2.8 \%, P=0.9)$, and other emergency departments ( $0 \%$ vs $1.6 \%, P=0.471)$.

\section{Discussion}

As an infectious disease transmission via respiratory droplet secretions or direct contact with the lesions, COVID-19 has caused a global pandemic in a short period, with a higher mortality rate than seasonal flu. [11-13]At present, isolating viruses from samples and testing for specific genes are the main diagnostic modality of choice. The COVID-19 genome mutations are continuously found in the course of the pandemic, and the transmission and virulence are also constantly changing with the mutations. Mankind is still in the exploratory stage of COVID-19. [14] The COVID-19 outbreak is severely affecting the health services system worldwide. $[15,16]$ COVID- 19 is accompanied by a variety of symptoms, $80 \%$ of infected patients even have no symptoms or mild symptoms. [17] Therefore, it is necessary for ED which is the frontline of hospitals to distinguish suspected infection patients more carefully. [18]In the current COVID19 situation, strict screening and careful admission are necessary. Therefore, all patients in the ED were subjected to epidemiological investigation and nucleic acid tests, and were subjected to isolation and protection. In the actual work process, it was found that for non-critical patients in ED, before the test results of in-room patients were confirmed in the ED, our next triage seemed to be limited, which may reduce the bed turnover rate in the ED.

This study found that LOS in the ED during the COVID-19 group was longer than the pre-COVID-19 group. Six hours is generally considered a reasonable level of ED stay. [19]The reasons for crowding in the ED can be divided into three aspects: input, throughput and output. For this study, there was no significant difference in the overall number of patients between pre-COVID-19 group and COVID-19 group. The crowding caused by the prolonged length of stay in the ED was mainly considered from two aspects: throughput and output. Several studies[20,21] have shown that waiting for hospitalization in ED is one of the main reasons for crowding and detention in ED. Fatovich et al. [22] considered that the root cause of ED overcrowding and retention lies in whether the hospital can provide enough inpatient beds. 
During the COVID-19, the hospitalization patients, SARS-Cov-2 nucleic acid tests, chest computerized tomography (CT) and other related examinations are needed to determine the next step, and the results of relevant examinations for patients in the ED cannot be obtained quickly, resulting in a certain delay in the evaluation and treatment of patients. During the outbreak of the COVID-19, some emergency medical staff respond to the call and arrived in the severely affected areas for assistance, which to a certain extent caused the shortage of ED staff and delayed the triage of patients.

Emergency internal medical patients usually have more complex conditions and have more complications. So, it takes more time for subspecialties to coordinate, and patients are likely to stay in the ED for a long time. During the course of admitting patients to specialties during the COVID-19 pandemic, subspecialities tend to put their emphasis on assessing patient epidemiological factors to prevent the spread of the epidemic, which makes it more difficult for patients to leave ED. In addition, during the COVID-19 pandemic, the workload of laboratory staff has increased to some extent, which directly affects the production speed of laboratory results. And the patient's condition evaluation not only depends on the symptoms but often also needs to combine the results of laboratory tests and radiographic data. Hospitalization or discharge should be decided after comprehensive consideration. The delay of laboratory results may reduce the bed turnover rate to a certain extent. [23]Trauma patients were the main reason for entering emergency surgery, and car accidents were one of the main reasons. Other surgical patients include patients with abdominal pain such as intestinal obstruction and appendicitis, and patients with aortic dissection, Etc. For surgical patients in urgent need of emergency surgery, the treatment in ED was mainly symptomatic treatment and maintaining vital signs, and emergency surgical treatment was the main part of the treatment. Such patients can still have access to the green channel during the COVID-19 pandemic. Instead of waiting for SARS-CoV-2 nucleic acid results, they can be treated with surgery in a timely way after a CT scan and rapid assessment by a COVID-19 assessment panel. Patients who were not undergoing emergency surgery during the COVID-19 pandemic had to wait in ED for SARS-CoV-2 nucleic acid results to be negative before they could be admitted to hospital. These patients could have been hospitalized only if a bed was available in the specialist ward. This may be an important reason for the prolonged LOS of emergency surgical patients in the ED.

Most of the patients first diagnosed in the emergency neurology department were cerebral infarction or spontaneous intracerebral hemorrhage $(\mathrm{ICH})$. Patients with severe intracerebral hemorrhage with surgical indications should be treated according to emergency surgical procedures. According to the recommended treatment strategies,[24-26] in addition to recombinant tissue plasminogen activator (rtPA), there are few effective treatments for ischemic stroke, but the therapeutic window of rT-PA is 4.5 hours, beyond which the risk of cerebral hemorrhage is significantly increased. So, emergency thrombolysis for eligible patients with cerebral infarction and symptomatic treatment for concurrent symptoms such as high fever, hyperglycemia, and dysphagia are generally completed in the stroke unit of the ED. After the end of emergency treatment, patients will be transferred to observation or hospitalization. In this process, it is enough to wait for the SARS-CoV-2 nucleic acid results of patients. This process of diagnosis and treatment may be the reason why the LOS of patients in the emergency neurology department was not prolonged during the COVID-19 pandemic period. 
Several studies revealed that[27-30] mortality increased is a common adverse consequence of ED crowding. However, this study shows that although the LOS of patients is longer in the ED during the COVID-19 pandemic period, the overall mortality rate does not change significantly. Generally, high mortality in the emergency room mainly includes acute heart disease, critically ill patients who need to be admitted to the Intensive Care Unit for monitoring and treatment, or patients who need emergency surgery. During the COVID-19 pandemic period, the hospital maintained the policy of emergency surgery and critically ill patients first and opened a green channel for critically ill patients. For example, critically ill patients with a low risk of COVID-19 infection should be admitted to subspecialized isolation wards for positive treatment, and be released when the results are negative. Patients requiring emergency surgery were reported to the medical office for surgical treatment and then isolated to a single ward for inspection results. Perhaps, the mortality is not increased during the COVID-19 pandemic period in ED due to the effective implementation of these measures.

Although the study suggested that the LOS in the ED had no significant adverse effect on mortality the overcrowded ED caused by the prolonged LOS in the ED still led to other adverse consequences, such as patients' unsatisfactory medical treatment, patients' privacy exposure, excessive work pressure of staff, medical disputes, Etc. The prolonged LOS in ED during the COVID-19 should be taken seriously. And staff, ED, hospitals and policymakers should work together to establish more effective triage mechanisms, such as increasing ED staff, improving ED rapid testing equipments, and increasing the number of isolation wards in each department in response to triage patients in ED during the COVID-19.

\section{Limitations}

There are some limitations to our study. First, this study was a single-center retrospective study, and the results could not reflect the situation of other hospitals in China. Second, in this study, there was no obvious change in mortality between the two groups. However, this result does not mean that the COVID19 pandemic has no impact on the prognosis of patients with prolonged LOS in ED. For example, some patients may die during treatment in specialized wards after leaving the ED. Unfortunately, we do not have data on patients after leaving the ED. Thirdly, it can be observed that LOS seems longer from January to March, and both emergency medicine and emergency neurology departments seem to have such a trend, which may be related to seasonal factors, more data need to be further collected to analyze the possible causes.

\section{Conclusion}

The COVID-19 pandemic was associated with a significant increase in the length of stay, which may lead to emergency department crowding. And the influence of the COVID-19 pandemic on patients in different emergency departments is different. There is no significant impact on the LOS of emergency neuropathy. Across departments, COVID-19 didn't have a significant impact on prognosis of ED patients.

\section{Abbreviations}


COVID-19

Coronavirus disease-2019

ED

emergency department

CDC

Chinese Center for Disease Control and Prevention

LOS

length of stay

IQRs

interquartile ranges

CT

computerized tomography

$\mathrm{ICH}$

intracerebral hemorrhage

rt-PA

recombinant tissue plasminogen activator

\section{Declarations}

Ethics approval and consent to participate: The work received ethical approval from the Medical Ethics Committee of the First Affiliated Hospital of Soochow University ((2021) Research Lot No. 317).

Participants were informed that their participation in the study was voluntary and that they had the right to withdraw from the study without providing any reason. All participants concent to participate. Informed consent was obtained from all participants. This retrospective cohort study was performed in accordance with the Declaration of Helsinki.

Competing interests: The authors declare they have no competing interests.

Consent to publish: Not applicable.

Availability of data and materials: The data generated and analyzed in this study may not be used publicly in order to protect participants' personal information, but may be obtained from corresponding authors upon reasonable request. (sz_xf@suda.edu.cn).

Competing interests: The authors declare that they have no conflict of interest.

Funding: The study received no special funding from any institution.

Authors' contributions: Fengbao Guo conceptualized and designed this study, conducting a statistical analysis of the data. Yan Qin wrote the original draft and provided references. Hailong Fu was responsible for data collection and investigation. Feng $\mathrm{Xu}$, the corresponding author and guarantor, 
introduced the purpose of this study to all participants. All authors contributed to the improvement of the paper and all authors approved the final manuscript.

Acknowledgements: We thank all the participants for their contributions, institutional support and peer reviewers for their helpful comments on this paper.

\section{References}

1. The species Severe acute respiratory syndrome-related coronavirus: classifying 2019-nCoV and naming it SARS-CoV-2. Nat Microbio/ 2020, 5(4):536-544.

2. Rafiq MF, Ahmed N, Khan AA: Serum electrolyte derangements in patients with traumatic brain injury. J Ayub Med Coll Abbottabad 2013, 25(1-2):162-164.

3. Breugelmans JG, Zucs P, Porten K, Broll S, Niedrig M, Ammon A, Krause G: SARS transmission and commercial aircraft. Emerg Infect Dis 2004, 10(8):1502-1503.

4. Hoot NR, Aronsky D: Systematic review of emergency department crowding: causes, effects, and solutions. Ann Emerg Med 2008, 52(2):126-136.

5. Moskop JC, Sklar DP, Geiderman JM, Schears RM, Bookman KJ: Emergency Department Crowding, Part 1-Concept, Causes, and Moral Consequences. Annals of Emergency Medicine 2009, 53(5):605611.

6. Asplin BR, Magid DJ, Rhodes KV, Solberg LI, Lurie N, Camargo CA: A conceptual model of emergency department crowding. Annals of Emergency Medicine 2003, 42(2):173-180.

7. Ahalt V, Argon NT, Ziya S, Strickler J, Mehrotra A: Comparison of emergency department crowding scores: a discrete-event simulation approach. Health Care Manag Sci 2018, 21(1):144-155.

8. Moskop JC, Geiderman JM, Marshall KD, McGreevy J, Derse AR, Bookman K, McGrath N, Iserson KV: Another Look at the Persistent Moral Problem of Emergency Department Crowding. Ann Emerg Med 2019, 74(3):357-364.

9. Bernstein SL, Asplin BR: Emergency department crowding: old problem, new solutions. Emerg Med Clin North Am 2006, 24(4):821-837.

10. Freund Y, Goulet H, Leblanc J, Bokobza J, Ray P, Maignan M, Guinemer S, Truchot J, Féral-Pierssens AL, Yordanov $Y$ et al: Effect of Systematic Physician Cross-checking on Reducing Adverse Events in the Emergency Department: The CHARMED Cluster Randomized Trial. JAMA Intern Med 2018, 178(6):812-819.

11. Arellanos-Soto D, Padilla-Rivas G, Ramos-Jimenez J, Galan-Huerta K, Lozano-Sepulveda S, MartinezAcuña N, Treviño-Garza C, Montes-de-Oca-Luna R, de-la OCM, Rivas-Estilla AM: Decline in influenza cases in Mexico after the implementation of public health measures for COVID-19. Sci Rep 2021, 11(1):10730.

12. Fowlkes A, Steffens A, Temte J, Lonardo SD, McHugh L, Martin K, Rubino H, Feist M, Davis C, Selzer C et al: Incidence of medically attended influenza during pandemic and post-pandemic seasons through the Influenza Incidence Surveillance Project, 2009-13. Lancet Respir Med 2015, 3(9):709-718. 
13. Herbstreit F, Overbeck M, Berger MM, Skarabis A, Brenner T, Schmidt K: Characteristics of Critically III Patients with COVID-19 Compared to Patients with Influenza-A Single Center Experience. J Clin Med 2021, 10(10).

14. Wiersinga WJ, Rhodes A, Cheng AC, Peacock SJ, Prescott HC: Pathophysiology, Transmission, Diagnosis, and Treatment of Coronavirus Disease 2019 (COVID-19): A Review. Jama 2020, 324(8):782-793.

15. Kim HS, Jang TC, Kim GM, Lee SH, Ko SH, Seo YW: Impact of the coronavirus disease 2019 outbreak on the transportation of patients requiring emergency care. Medicine (Baltimore) 2020, 99(50):e23446.

16. Emanuel EJ, Persad G, Upshur R, Thome B, Parker M, Glickman A, Zhang C, Boyle C, Smith M, Phillips JP: Fair Allocation of Scarce Medical Resources in the Time of Covid-19. N Engl J Med 2020, 382(21):2049-2055.

17. Wu Z, McGoogan JM: Characteristics of and Important Lessons From the Coronavirus Disease 2019 (COVID-19) Outbreak in China: Summary of a Report of 72314 Cases From the Chinese Center for Disease Control and Prevention. Jama 2020, 323(13):1239-1242.

18. Oh YJ, Kim GM, Ko SH, Seo YW, Lee SH, Jang TC: Effects of dynamic response to coronavirus disease outbreak in a regional emergency medical center: A retrospective study. Medicine (Baltimore) 2021, 100(28):e26634.

19. Henneman PL, Nathanson BH, Li H, Smithline HA, Blank FS, Santoro JP, Maynard AM, Provost DA, Henneman EA: Emergency department patients who stay more than 6 hours contribute to crowding. J Emerg Med 2010, 39(1):105-112.

20. Richardson DB, Mountain D: Myths versus facts in emergency department overcrowding and hospital access block. Med J Aust 2009, 190(7):369-374.

21. Hillier DF, Parry GJ, Shannon MW, Stack AM: The effect of hospital bed occupancy on throughput in the pediatric emergency department. Ann Emerg Med 2009, 53(6):767-776.e763.

22. Fatovich DM, Hughes G, McCarthy SM: Access block: it's all about available beds. Med J Aust 2009, 190(7):362-363.

23. Nuñez-Argote L, Baker DP, Jones AP: Initial Clinical Laboratory Response to COVID-19: A Survey of Medical Laboratory Professionals. Lab Med 2021, 52(4):e115-e124.

24. Feske SK: Thrombolytic therapy of acute stroke. Circulation 2012, 125(21):2662-2666.

25. Shinozuka K, Dailey T, Tajiri N, Ishikawa H, Kim DW, Pabon M, Acosta S, Kaneko Y, Borlongan CV: Stem Cells for Neurovascular Repair in Stroke. J Stem Cell Res Ther 2013, 4(4):12912.

26. Chen $C$, Wang Y, Yang GY: Stem cell-mediated gene delivering for the treatment of cerebral ischemia: progress and prospectives. Curr Drug Targets 2013, 14(1):81-89.

27. Miró O, Antonio MT, Jiménez S, De Dios A, Sánchez M, Borrás A, Millá J: Decreased health care quality associated with emergency department overcrowding. Eur J Emerg Med 1999, 6(2):105-107. 
28. Richardson DB: Increase in patient mortality at 10 days associated with emergency department overcrowding. Med J Aust 2006, 184(5):213-216.

29. Sprivulis PC, Da Silva JA, Jacobs IG, Frazer AR, Jelinek GA: The association between hospital overcrowding and mortality among patients admitted via Western Australian emergency departments. Med J Aust 2006, 184(5):208-212.

30. Begley CE, Chang Y, Wood RC, Weltge A: Emergency department diversion and trauma mortality: evidence from houston, Texas. J Trauma 2004, 57(6):1260-1265.

\section{Figures}

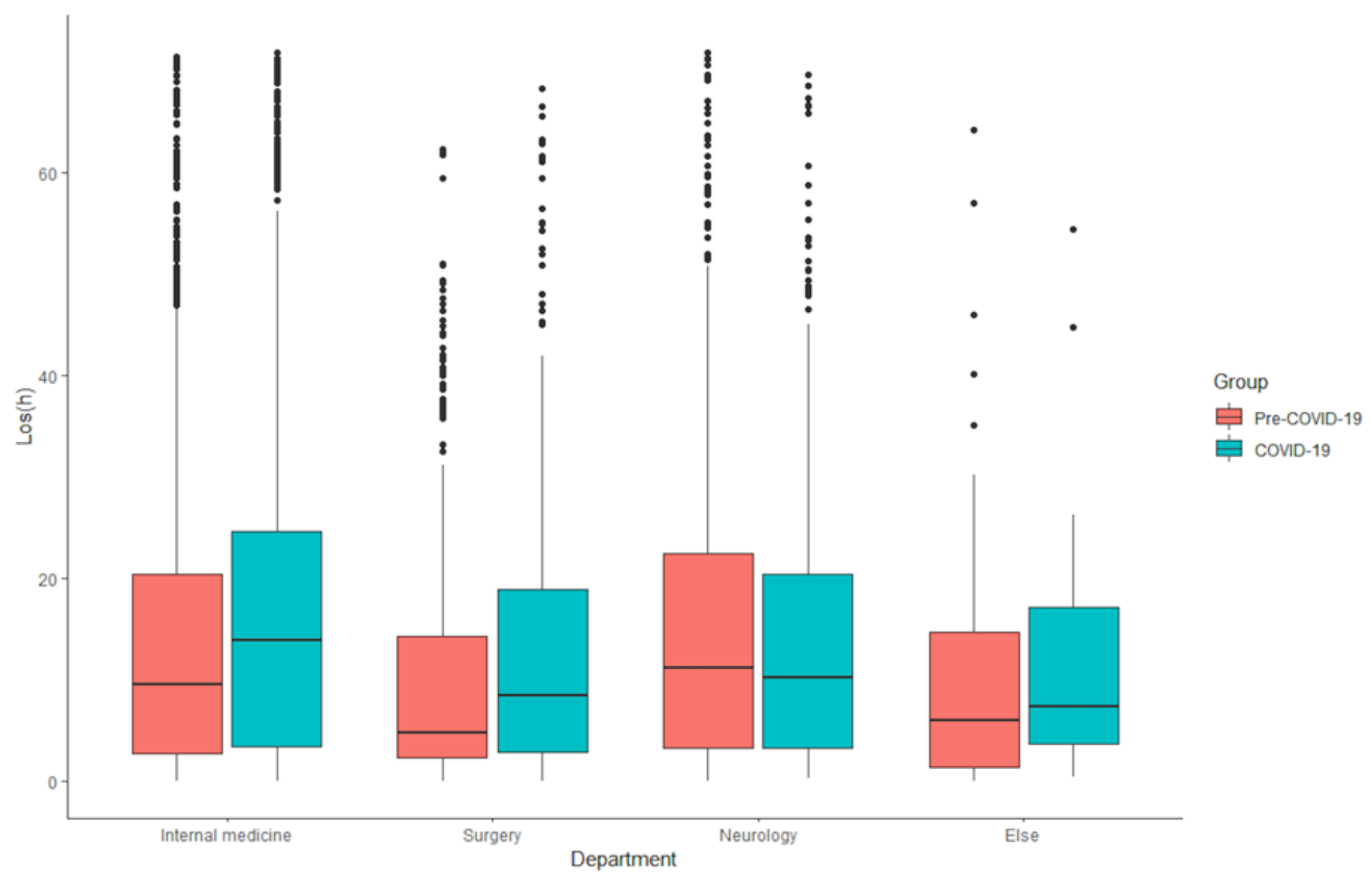

Figure 1

Box plot of LOS in different departments in Pre-COVID-19 group vs COVID-19 group 

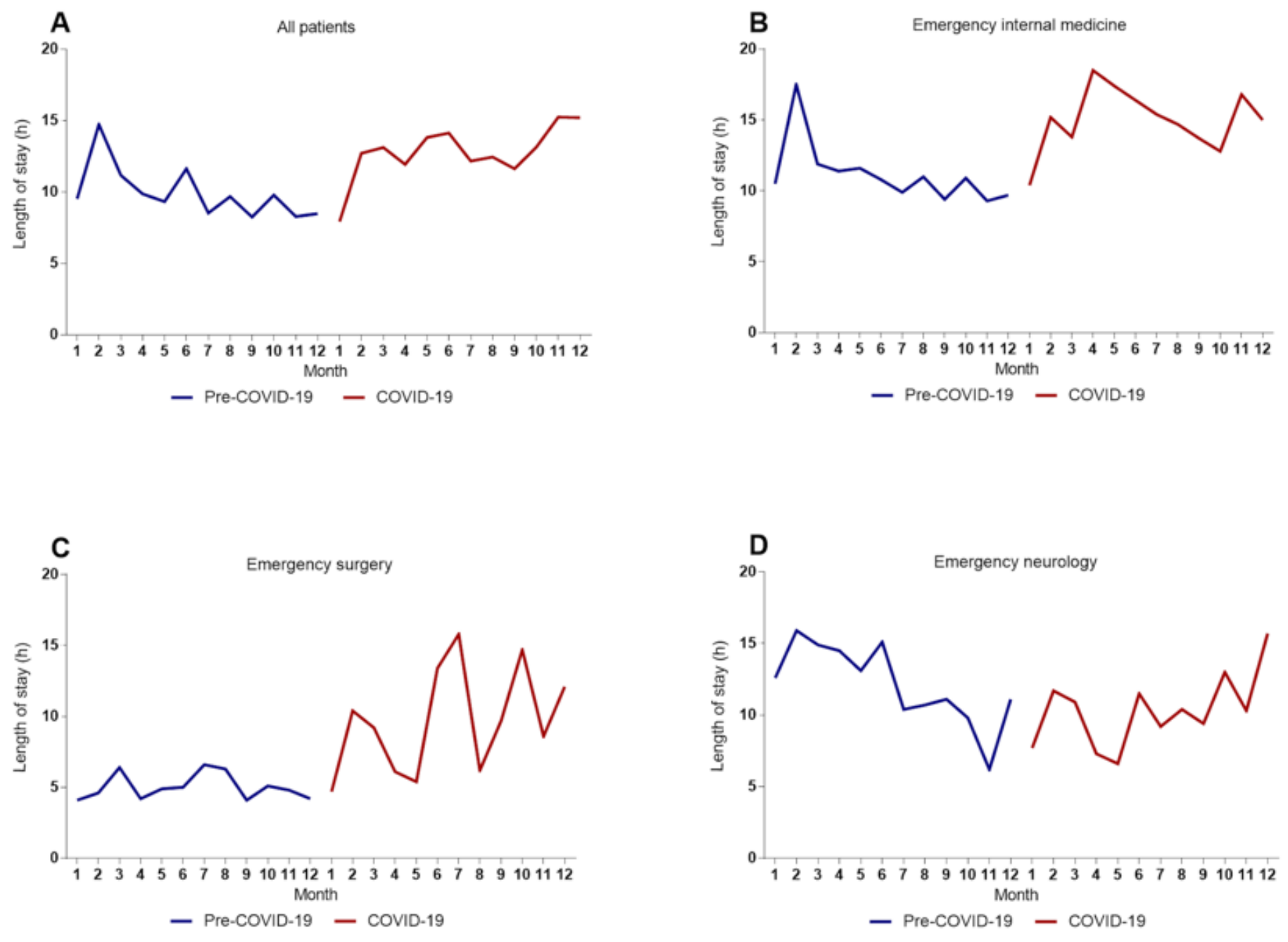

Figure 2

Trend of LOS in pre-COVID-19 group and COVID-19 group A The trend of LOS in all patients B The trend of LOS in emergency internal medicine $\mathbf{C}$ The trend of LOS in emergency surgery $\mathbf{D}$ The trend of LOS in emergency neurology 